Supply Management Research 
Ronald Bogaschewsky · Michael Eßig

Rainer Lasch - Wolfgang Stölzle

\section{Supply Management Research}

Aktuelle Forschungsergebnisse 2012

Springer Gabler 


\section{Herausgeber}

Prof. Dr. Ronald Bogaschewsky

Universität Würzburg

Deutschland

Prof. Dr. Michael Eßig

Universität der Bundeswehr München

Deutschland
Prof. Dr. Rainer Lasch

Universität Dresden

Deutschland

Prof. Dr. Wolfgang Stölzle

Universität St. Gallen

Schweiz

Die Deutsche Nationalbibliothek verzeichnet diese Publikation in der Deutschen Nationalbibliografie; detaillierte bibliografische Daten sind im Internet über http://dnb.d-nb.de abrufbar.

\section{Springer Gabler}

(c) Gabler Verlag | Springer Fachmedien Wiesbaden GmbH 2012

Das Werk einschließlich aller seiner Teile ist urheberrechtlich geschützt. Jede Verwertung, die nicht ausdrücklich vom Urheberrechtsgesetz zugelassen ist, bedarf der vorherigen Zustimmung des Verlags. Das gilt insbesondere für Vervielfältigungen, Bearbeitungen, Übersetzungen, Mikroverfilmungen und die Einspeicherung und Verarbeitung in elektronischen Systemen.

Die Wiedergabe von Gebrauchsnamen, Handelsnamen, Warenbezeichnungen usw. in diesem Werk berechtigt auch ohne besondere Kennzeichnung nicht zu der Annahme, dass solche Namen im Sinne der Warenzeichen- und Markenschutz-Gesetzgebung als frei zu betrachten wären und daher von jedermann benutzt werden dürften.

Einbandentwurf: KünkelLopka GmbH, Heidelberg

Gedruckt auf säurefreiem und chlorfrei gebleichtem Papier

Springer Gabler ist eine Marke von Springer DE. Springer DE ist Teil der Fachverlagsgruppe Springer Science+Business Media

www.springer-gabler.de 


\section{Geleitwort}

Die „Practice meets Science“-Initiative des Bundesverbandes Materialwirtschaft, Einkauf und Logistik e.V. (BME) steht für den konstruktiven, offenen Austausch zwischen Praktikern und Wissenschaftlern. Dabei unterstützt der Verband aktiv das Aufspüren von Trends und Innovationen, das Erarbeiten von Erfolgsansätzen, das Vermitteln von Erprobtem und das Vernetzen interessierter Menschen und ihrer Ideen. Für den Austausch bietet der BME mit seiner fast 60-jährigen Erfahrung seinen rund 8.000 Mitgliedern und einer breiten Fachöffentlichkeit eine ideale Plattform.

Die wissenschaftliche Auseinandersetzung mit den Themen Beschaffung und Logistik, verbunden mit der Förderung des wissenschaftlichen Nachwuchses, ist eine wichtige Säule des BME. Hier gilt es, Anreize für Arbeiten zum Thema Supply Management zu setzen. Seit 1988 zeichnet der Verband Verfasser der besten Habilitationsschriften und Dissertationen mit dem „BME-Wissenschaftspreis“ aus. Herausragende Studienabschlussarbeiten werden seit 2003 mit den „BME-Hochschulpreis für Beschaffung und Logistik“ prämiert. Der „BME-Preis Trendscouting“ für Abschlussarbeiten zum Themenbereich Logistik wird seit 2007 vergeben.

Mit seiner BME-Buchreihe „Advanced Studies in Supply Management" macht der Verband wichtige wissenschaftliche Erkenntnisse rund um aktuelle und vieldiskutierte Managementmethoden transparent und stellt diese einer breiten Öffentlichkeit zur Verfügung. Ich freue mich sehr, dass auch der fünfte Band wieder interessante Lösungsansätze für aktuelle Herausforderungen aufzeigt. Beispielhaft nenne ich hier Aufsätze zur Entwicklung innovativer Supply-Chain-Management-Konzepte, zum Risk Pooling in Business Logistics und zur Flexibilisierung von Frachtraten in langfristigen Luftfrachtverträgen. Interessante Lösungsansätze für Praktiker in den Unternehmen bieten u. a. Beiträge zur nachhaltigen Rohstoffbeschaffung, zum Risikomanagement in Beschaffung und Distribution sowie zur Verteilung von Koalitionsgewinnen in Beschaffungskooperationen.

Mein herzlicher Dank gilt den Autoren für ihre Beiträge sowie insbesondere den Professoren Ronald Bogaschewsky, Michael Eßig, Rainer Lasch und Wolfgang Stölzle für ihre langjährige fachliche Unterstützung und ihr großes Engagement.

Frankfurt, im März 2012

Dr. Holger Hildebrandt

Hauptgeschäftsführer Bundesverband Materialwirtschaft, Einkauf und Logistik e.V. 


\section{Vorwort}

Das vorliegende Buch ist der fünfte Band der im Jahre 2008 gestarteten Buchreihe "Advanced Studies in Supply Management", in der jährlich die wissenschaftlichen Fortschritte in diesem Forschungsfeld dargelegt werden. Zugleich handelt es sich um den Tagungsband des „5. Wissenschaftlichen Symposiums Supply Management“, das im Frühjahr 2012 durchgeführt wurde. Diese jährlich ausgerichtete Tagung wird vom Bundesverband Materialwirtschaft, Einkauf und Logistik e. V. (BME) veranstaltet, der auch die Buchreihe herausgibt. Inhaltlich verantwortlich für die Durchführung der Symposien und die hieraus resultierenden Schriften ist der Wissenschaftliche Beirat des Bundesvorstands des BME.

Die hohe Bedeutung der Bereiche Beschaffung, Einkauf, Materialwirtschaft, Logistik und Supply Chain Management spiegelt sich in den zunehmend intensiven Forschungsanstrengungen der - theoriegeleiteten wie der anwendungsnahen - Wissenschaft wider. Mit dem Wissenschaftlichen Symposium Supply Management konnte hierfür eine adäquate und inzwischen etablierte Diskussions- und Präsentationsplattform im europäischen Raum geschaffen werden.

Alle in diesem Band aufgenommenen, in primär wissenschaftlich und stärker anwendungsnah differenzierten Beiträge mussten sich einem Double-blind-Review-Verfahren unterziehen und wurden von unabhängigen Gutachtern eingehend geprüft. Diesen gilt unser Dank für die gewissenhafte Erstellung der Gutachten und die auf diesem Wege bereitgestellten Verbesserungsvorschläge für die Beiträge. Zahlreiche Einreichungen wurden abgelehnt, da sie den rigorosen Ansprüchen der Gutachter nicht genügten. Aufgenommen wurden zudem die drei Arbeiten, die sich für das Finale des „BME-Wissenschaftspreises“ aus einer großen Anzahl Einreichungen qualifizieren konnten. Der Jury des „BME-Wissenschaftspreises“ gilt ebenfalls unser Dank für die geleisteten Begutachtungen. Ein herzliches Dankeschön geht an Ulrike Müller, die wiederum in höchstem Maße zuverlässig und sehr sorgfältig das gesamte Projekt Wissenschaftliches Symposium samt Tagungsband betreute.

Es war und ist erklärtes Ziel, ausschließlich exzellente Forschungsergebnisse sowie innovative Beiträge mit hoher Praxisrelevanz auf dem Wissenschaftlichen Symposium zu präsentieren und im Tagungsband zu publizieren. Der vorliegende Band zeigt die große Breite und erhebliche Tiefe der Erkenntnisse im Bereich Supply Management auf. Es ist dem Wissenschaftlichen Beirat und dem BME ein besonderes Anliegen, diese Arbeiten weiterhin intensiv zu fördern.

Im Januar 2012

Prof. Dr. Ronald Bogaschewsky, Würzburg

Prof. Dr. Rainer Lasch, Dresden
Prof. Dr. Michael Eßig, München

Prof. Dr. Wolfgang Stölzle, St. Gallen 


\section{Inhaltsverzeichnis}

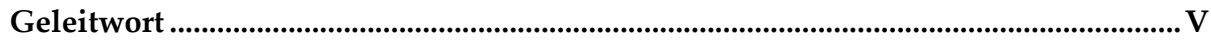

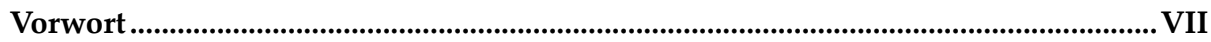

Teil A Wissenschaftliche Forschungsbeiträge ..............................................1

Multi-Period Supplier Selection and Supplier Development under Dynamic and

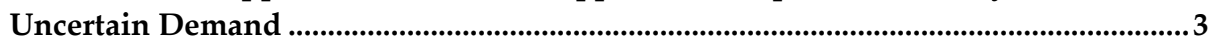

Frank Meisel

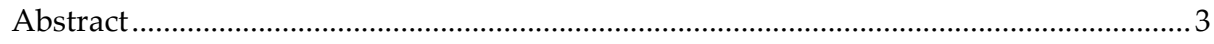

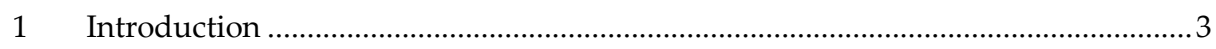

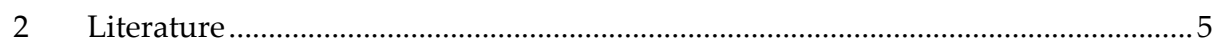

2.1 Studies on Supplier Evaluation and Selection.................................................

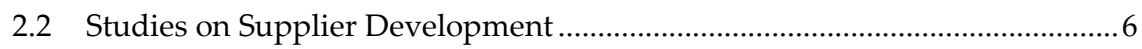

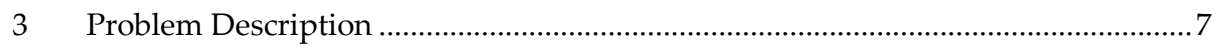

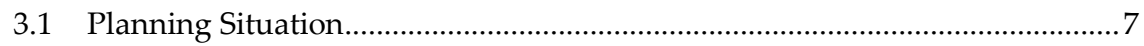

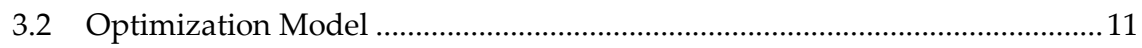

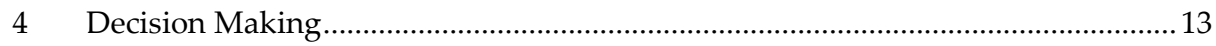

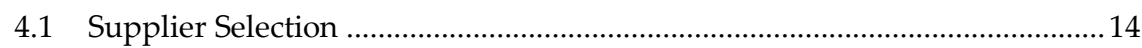

4.2 Scheduling of Development Projects............................................................... 15

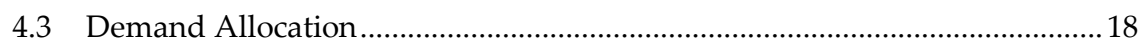

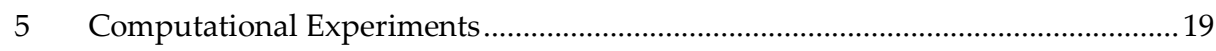

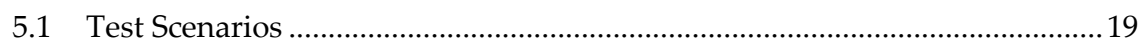

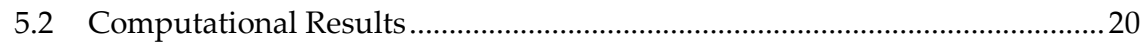

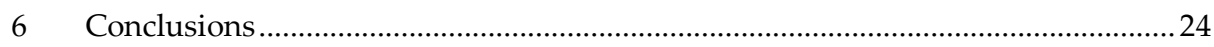

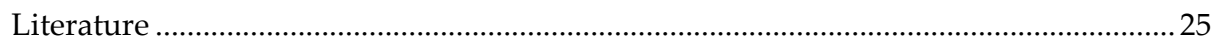

Performance Control in Buyer-Supplier-Relationships - The Design and Use of Formal Management Control Systems...................................................................................29

Konstantin Gebert

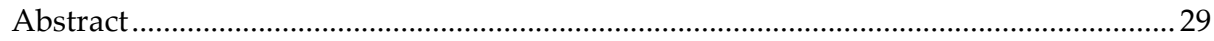

1 Performance control in buyer-supplier relationships - A burning issue in today's business environments................................................................................. 30

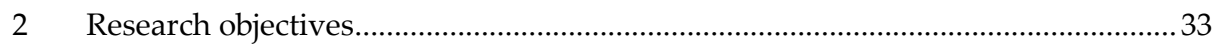

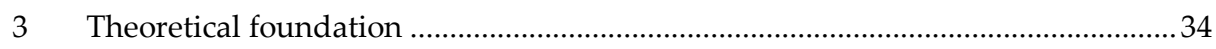

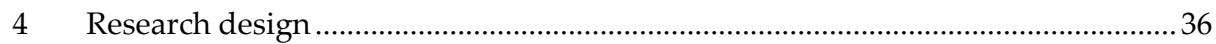


$5 \quad$ Findings 39

5.1 Contributions to practice and managerial implications................................43

5.2 Contributions to research and scientific implications .....................................4 44

Literature

Procurement Value Added - Ein Ansatz zur wertorientierten Messung des

Beschaffungserfolgs

Daniel Maucher, Wolfgang Stölzle und Erik Hofmann

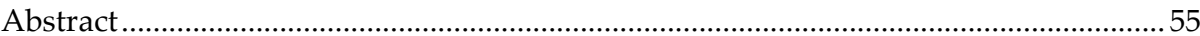

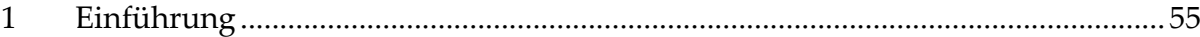

2 Einfluss der Beschaffung auf den Unternehmenserfolg........................................57

3 Einordnung der wertorientierten Erfolgsmessung in das Beschaffungscontrolling

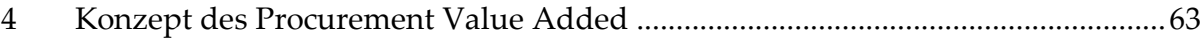

5 Exemplarische Anwendung des Procurement Value Added...................................67

$6 \quad$ Zusammenfassung, Diskussion und Ausblick .......................................................... 72

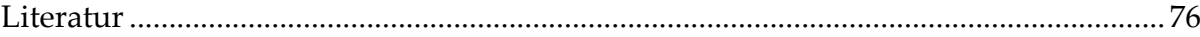

Innovative Supply Risk Management .........................................................................................79

Petra Hoffmann

Abstract 79

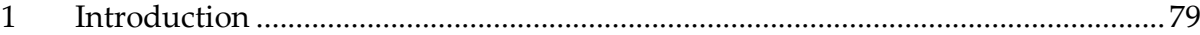

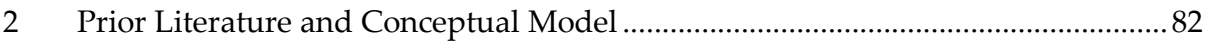

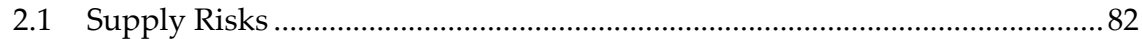

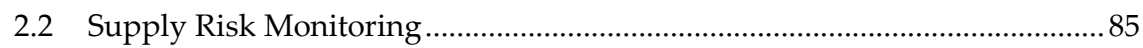

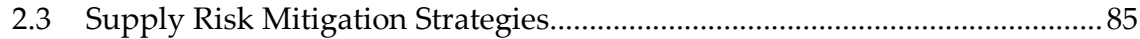

2.4 Conceptual Model: a Supply Risk Management Framework .........................86

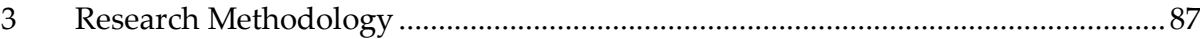

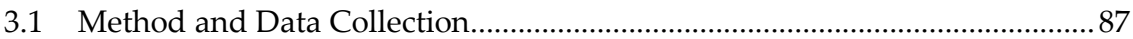

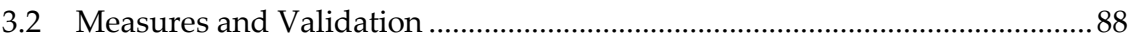

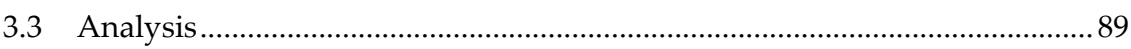

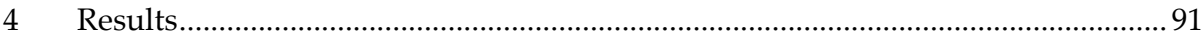

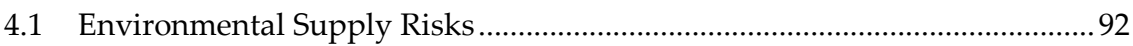

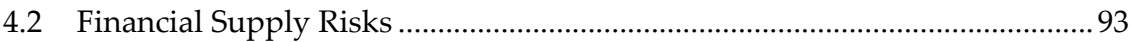

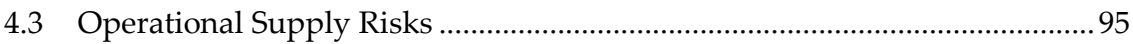

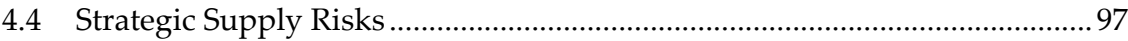

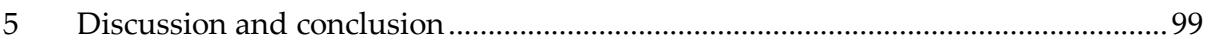

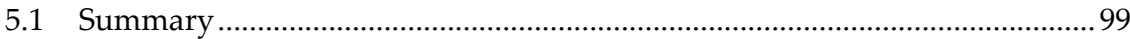

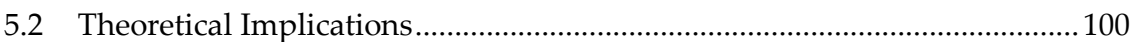

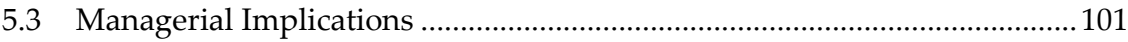


6 Research Limitations and Future Research. 101

Literature 102

Die Entwicklung innovativer Supply-Chain-Management-Konzepte Bedarf und Prozessmodell 105

Ingrid Göpfert und Wanja Wellbrock

Abstract.

1 Problemstellung 105

2 Die Notwendigkeit innovativer Supply-Chain-Management-Konzepte. 106

2.1 Ziele des Supply Chain Management

2.2 Zielerreichungsgrad der bestehenden Supply-Chain-ManagementKonzepte 110

2.3 Zukünftige Herausforderungen an das Supply Chain Management

3 Der Entwicklungsprozess innovativer Supply-Chain-Management-Konzepte.. 115

3.1 Merkmale und Charakteristika von Supply-Chain-ManagementInnovationen

3.2 Entwurf eines Entwicklungsprozessmodells ................................................. 116

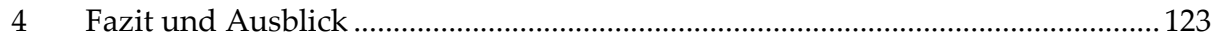

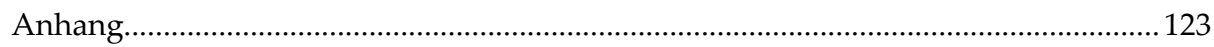

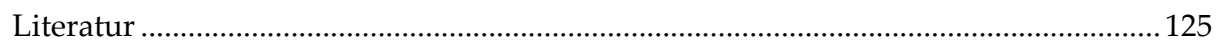

Towards a social exchange theory perspective on preferred customership concept and practice

Holger Schiele, Jasper Veldman, Lisa Hüttinger und Niels Pulles

Abstract

1 Introduction: Accessing suppliers in oligopolistic B2B markets.....

2 The framework of Preferred Customership in light of social exchange theory ..135

2.1 Customer attractiveness: precondition for starting a relationship ..............136

2.2 Supplier satisfaction: Customers meeting or exceeding expectations .........138

2.3 Preferred Customer Status: achieving relational rents.................................139

2.4 Closing the circle: From Preferred Customer Status to customer

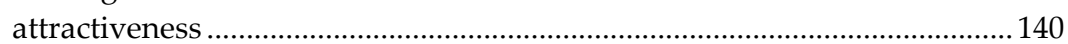

3 Customer attractiveness and supplier satisfaction in practice .............................. 142

3.1 World-Café: Incorporating practice into academic research ........................ 143

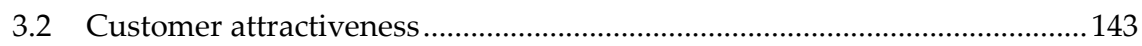

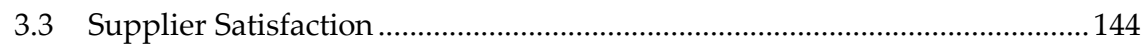

3.4 Preferred customer strategy ......................................................................... 144

4 Conclusion and research agenda: establishing a virtuous circle in order to generate competitive advantages ............................................................................ 145

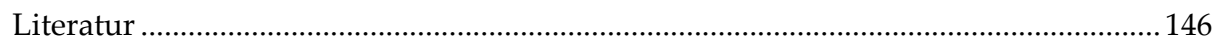


A Framework for Risk Pooling in Business Logistics

Gerald Oeser

Abstract

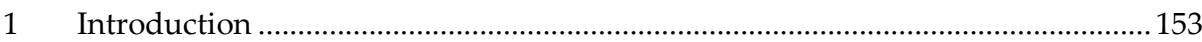

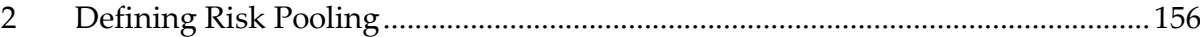

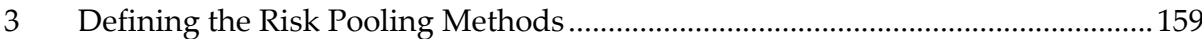

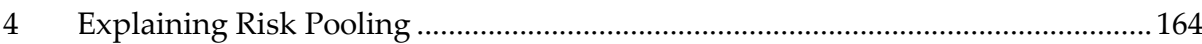

5 Placing Risk Pooling in the Supply Chain, Business Logistics,

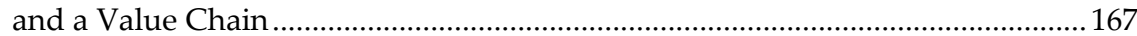

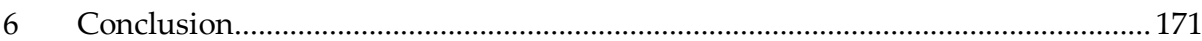

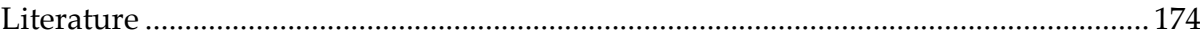

Flexibilisierung von Frachtraten in langfristigen Luftfrachtverträgen Umsetzung in Form einer Indexkopplung.......................................................................195

Ralf Elbert und Cora Bogusch

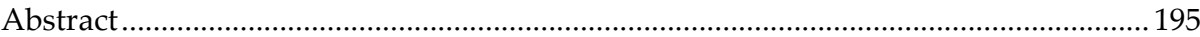

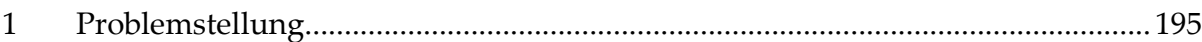

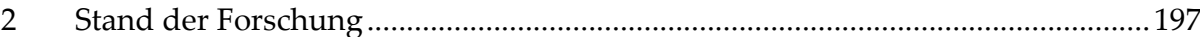

2.1 Theoretische Möglichkeiten der Preisfestlegung in langfristigen

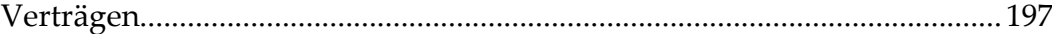

2.2 Anwendung der Preisflexibilisierung in der Praxis ......................................198

2.3 Gestaltungsoptionen einer Preisflexibilisierung als Indexkopplung

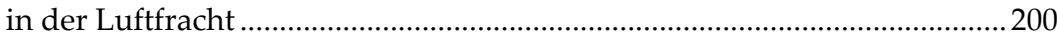

3 Fallstudie - Analyse historischer Vertragsdaten................................................202

3.1 Forschungsmethode und Datenkorpus..........................................................202

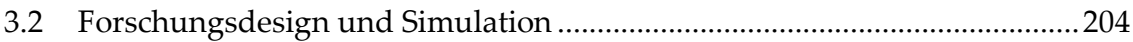

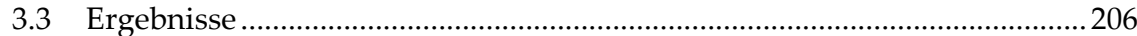

3.4 Analyse und Diskussion der Ergebnisse....................................................207

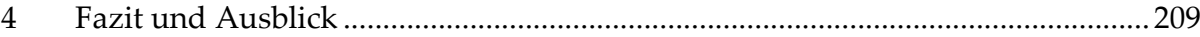

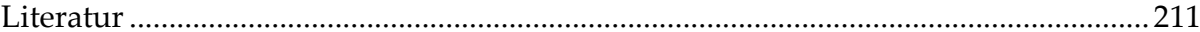

Teil B Anwendungsorientierte Beiträge.......................................................215

Entwicklung eines Gestaltungsmodells zur nachhaltigen Rohstoffbeschaffung

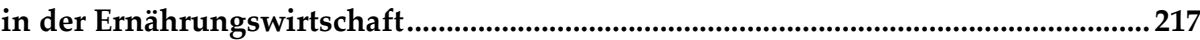

Therese Kirsch, Wolfgang Buchholz und Petra Teitscheid

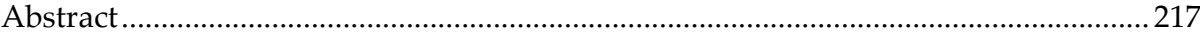

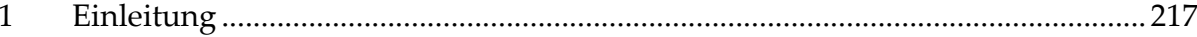

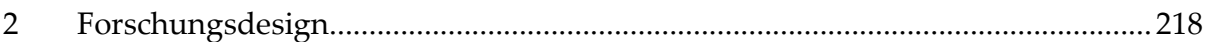

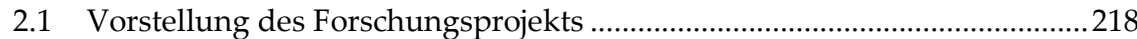

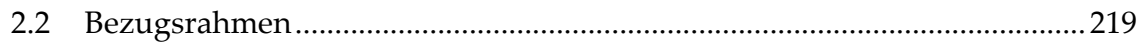




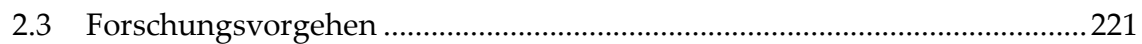

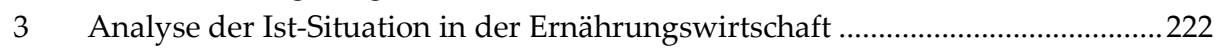

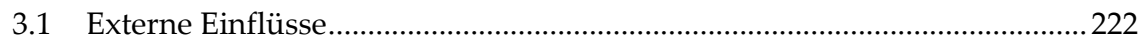

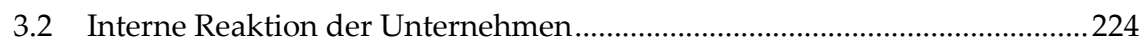

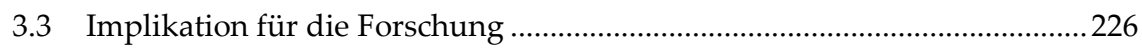

4 Gestaltungsmodell und Instrumente zur Umsetzung einer nachhaltigen

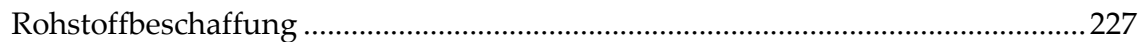

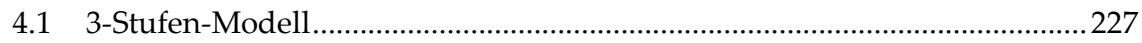

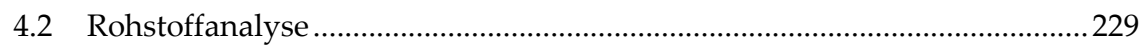

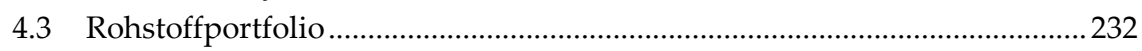

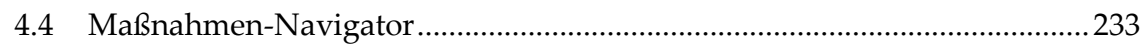

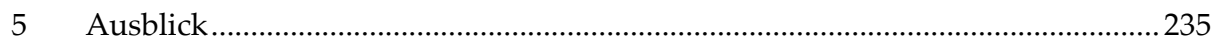

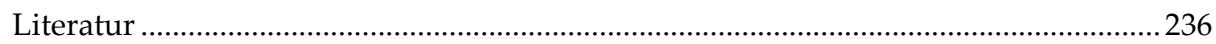

Verteilung von Koalitionsgewinnen in horizontalen Beschaffungskooperationen

Dirk Sackmann und Alexandra Rittmann

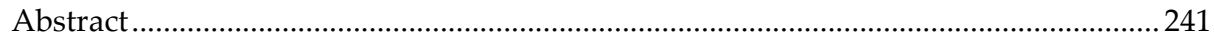

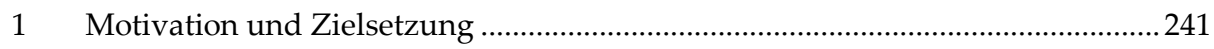

2 Spieltheoretische Lösungskonzepte zur Verteilung von Koalitionsgewinnen ...242

3 Basisanforderungen, Axiome und Lösungskonzepte.............................................243

3.1 Der Kern ............................................................................................... 244

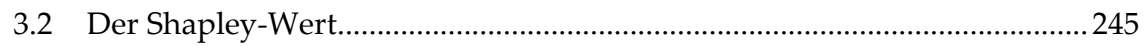

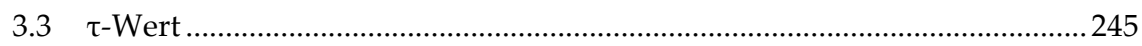

4 Anwendungsszenario und Auswertungen............................................................246

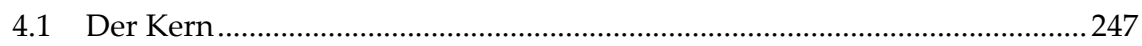

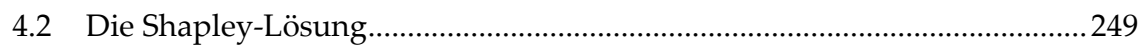

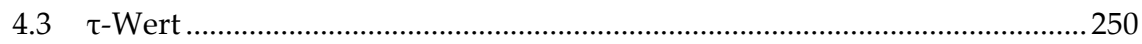

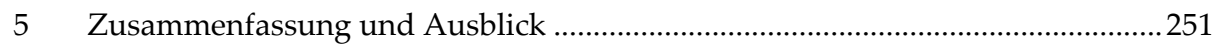

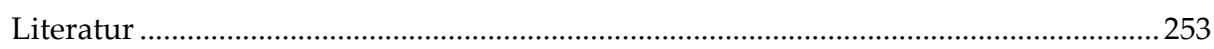

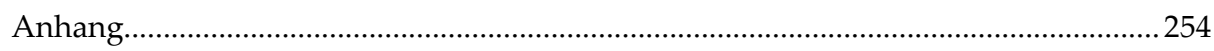

Risikomanagement in Beschaffung und Distribution - Entwicklung eines technologieorientierten Maßnahmenrasters zur Schadenverhütung im Transport.........255

Alexander C. H. Skorna, Philipp Hirsbrunner und Christoph Bode

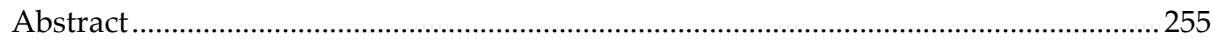

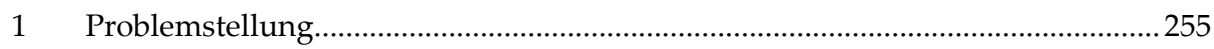

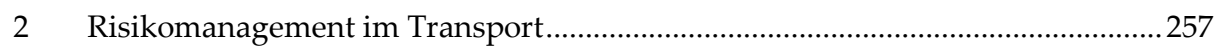

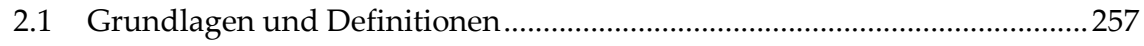

2.2 Identifikation von Transportrisiken und Schadenursachen..........................260 
2.3 Organisatorische Maßnahmen zur Schadenverhütung .264

2.4 Technologieorientierte Maßnahmen zur Schadenverhütung .......................2265

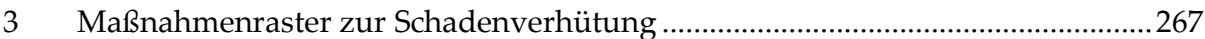

3.1 Methodik zur Bewertung des Technologieportfolios..................................267

3.2 Entwicklung des Maßnahmenrasters .......................................................268

3.3 Expertenbefragung zu Schadenverhütungspotenzialen technologieorientierter Maßnahmen

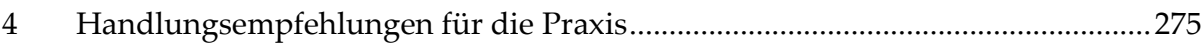

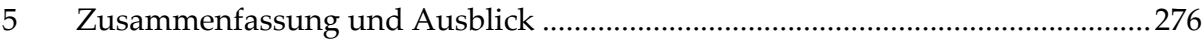

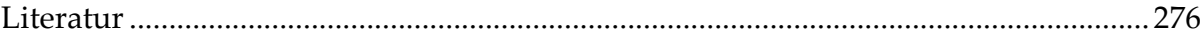

Intermodale Transportkettenplanung in logistischen Netzwerken ..............................281

Dirk Sackmann

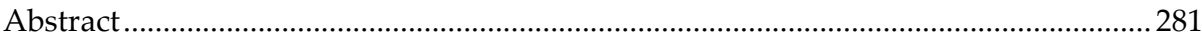

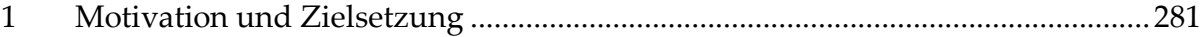

2 Einordnung und Abgrenzung der Problemstellung ............................................2. 283

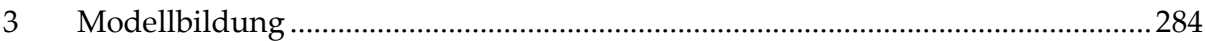

3.1 Rahmenbedingungen der Modellbildung ....................................................2 284

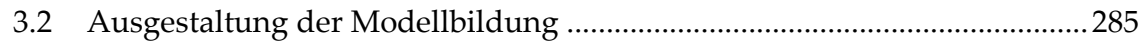

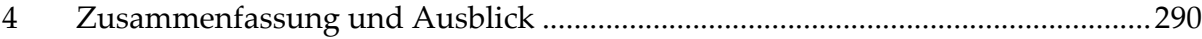

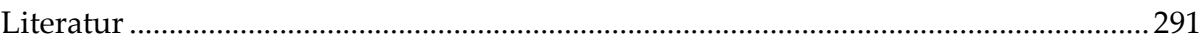

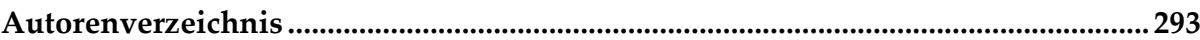

